ORIGINAL ARTICLE

\title{
Interleukin 4 induces apoptosis of acute myeloid leukemia cells in a Stat6-dependent manner
}

\author{
P Peña-Martínez ${ }^{1}$, M Eriksson ${ }^{1}$, R Ramakrishnan ${ }^{1}$, M Chapellier ${ }^{1}$, C Högberg ${ }^{1}$, C Orsmark-Pietras ${ }^{1}$, J Richter ${ }^{2,3}$, A Andersson ${ }^{1}$, \\ T Fioretos ${ }^{1}$ and $M$ Järås ${ }^{1}$
}

Cytokines provide signals that regulate immature normal and acute myeloid leukemia (AML) cells in the bone marrow microenvironment. We here identify interleukin 4 (IL4) as a selective inhibitor of AML cell growth and survival in a cytokine screen using fluorescently labeled AML cells. RNA-sequencing of the AML cells revealed an IL4-induced upregulation of Stat6 target genes and enrichment of apoptosis-related gene expression signatures. Consistent with these findings, we found that IL4 stimulation of AML cells induced Stat6 phosphorylation and that disruption of Stat6 using CRISPR/Cas9-genetic engineering rendered cells partially resistant to IL4-induced apoptosis. To evaluate whether IL4 inhibits AML cells in vivo, we expressed IL4 ectopically in AML cells transplanted into mice and also injected IL4 into leukemic mice; both strategies resulted in the suppression of the leukemia cell burden and increased survival. Notably, IL4 exposure caused reduced growth and survival of primary AML CD $34^{+} C D 38^{-}$patient cells from several genetic subtypes of AML, whereas normal stem and progenitor cells were less affected. The IL4-induced apoptosis of AML cells was linked to Caspase-3 activation. Our results demonstrate that IL4 selectively induces apoptosis of AML cells in a Stat6-dependent manner-findings that may translate into new therapeutic opportunities in AML.

Leukemia (2018) 32, 588-596; doi:10.1038/leu.2017.261

\section{INTRODUCTION}

Acute myeloid leukemia (AML) is a fatal disorder characterized by an accumulation of immature myeloid blasts in the bone marrow. Current treatment protocols have improved only modestly for the past 30 years and AML is associated with a poor overall survival, especially among the elderly (5-year overall survival of $\sim 20 \%$ in patients 60 or more years old). ${ }^{1,2}$

With increased understanding of how immature normal and leukemic cells interact with the microenvironment, new possibilities for intervening selectively against malignant cells are emerging. ${ }^{3}$ Successful examples in preclinical AML models comprise targeting of the leukemia microenvironment by CXCR4 inhibition to overcome resistance to kinase inhibitors and chemotherapy, ${ }^{4}$ and blocking of CD47 on AML cells to induce preferential phagocytosis of leukemia cells. ${ }^{5}$ In addition to cell-cell interactions, cytokines such as interleukin 6 (IL6) and tumor necrosis factor-a have been shown to positively regulate leukemia stem cells (LSCs), ${ }^{6,7}$ a self-renewing cell population capable of initiating and propagating leukemia. ${ }^{8}$ Consistent with IL1 being a positive regulator of LSCs, we and others recently showed that blocking IL1 signaling inhibits LSCs. ${ }^{9-11}$ However, little is known about cytokines that negatively affect LSC growth and survival, and whether such cytokines can be utilized for novel treatment approaches in AML.

To identify endogenous suppressors of immature AML cells, we performed a cytokine screen and identified IL4 as a negative regulator of primitive AML cells. IL4 induced apoptosis of AML cells in a Stat6-dependent manner, thus revealing a previously unrecognized role of IL4 as an inhibitor of the growth and survival of primitive AML cells.

\section{MATERIALS AND METHODS}

Murine leukemia model

Murine MLL-AF9 leukemia cells were propagated and harvested essentially as described before. ${ }^{12,13}$ For details on the in vivo model and culture conditions, the colony-forming assay and the bone marrow-homing assay, see Supplementary Information.

\section{Cytokine screen}

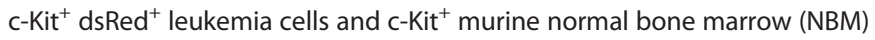
cells were mixed and seeded into $96-$ well plates. The cells were cultured in a serum-free expansion medium (Stemspan, StemCell Technologies, Vancouver, BC, Canada) containing $1 \%$ penicillin/streptomycin supplemented with one cytokine $(100 \mathrm{ng} / \mathrm{ml})$ condition per well (114 murine cytokines; purchased from Prospec, East Brunswick, NJ, USA; Peprotech, Rocky Hill, NJ, USA; and R\&D Systems, Minneapolis, MN, USA; listed in Supplementary Table 1). The cells were incubated for $72 \mathrm{~h}$ and cell numbers were determined by flow cytometry using CountBright beads (Life Technologies, Carlsbad, CA, USA).

\section{Flow cytometric analysis and cell sorting}

The flow cytometric analyses were performed using a FACS Canto II (BD Biosciences, San Jose, CA, USA) or a FACS LSRFortessa (BD Biosciences), and cell sorting was performed using a FACS Aria II (BD Biosciences). For details on the cell cycle analysis, apoptosis analysis and antibodies used, see Supplementary Information.

\section{Competitive bone marrow transplantations}

C57BL/6, B6SJL (both from Taconic, Hudson, NY, USA) and C57BL/6 $\times$ B6SJL (in-house breeding) were used for the experiments. For details on the experimental design, see Supplementary Information. 
Viral vectors

For details on experiments involving the murine stem cell virus gammaretroviral vector coexpressing a $\mathrm{m} / \mathrm{L} 4 \mathrm{CDNA}$, and lentiviral vectors expressing Cas9 ${ }^{+}$or a Stat6 single-guide RNA (sgRNA), see Supplementary Information.

\section{RNA-sequencing analysis}

Global gene expression profiling was performed on c-Kit ${ }^{+}$leukemia cells and $\mathrm{c}-\mathrm{Kit}^{+}$NBM cells cultured for $18 \mathrm{~h}$, as well as freshly isolated c-Kit ${ }^{+}$ leukemia cells. For details on the RNA extraction, library preparation and analysis, see Supplementary Information. Raw data and normalized gene expression data are available in the Gene Expression Omnibus database under accession number GSE79068.

\section{Human AML and NBM samples}

Mononuclear cells from AML patients and healthy volunteers were obtained after informed consent using ethical permits approved by the Regional Ethics Committee at Lund University. Mononuclear cells were separated using Lymphoprep (Axis Shield PoC AS, Dundee, UK), and CD34 ${ }^{+}$ cells were enriched using the human CD34 MicroBead Kit (Miltenyi Biotec, Bergisch Gladbach, Germany). For culture conditions, see Supplementary Information. Patient data are summarized in Supplementary Table 2.

\section{Statistical analyses}

Prism 6 (Graphpad Software, La Jolla, CA, USA) was used for statistical analyses including Student's $t$-tests, analyses of variance, linear regressions and Kaplan-Meier survival analysis. Significance is depicted with asterisks: ${ }^{*} P<0.05$, ${ }^{* *} P<0.01,{ }^{* * *} P<0.001,{ }^{* * *} P<0.0001$. Data are presented as mean \pm s.d.'s.

\section{RESULTS}

Cytokine screening identifies IL4 as an inhibitor of primitive AML cells

To identify cytokines acting as selective negative regulators of primitive AML cells affecting their growth and survival, we performed an in vitro cytokine screen using dsRed ${ }^{+} \mathrm{c}-\mathrm{Kit}^{+}$murine AML cells mixed with c-Kit ${ }^{+}$NBM cells, allowing for fluorescencebased separation of the two cell types (Figure 1a). The dsRed ${ }^{+}$ leukemia cells express the MLL-AF9 fusion gene and we have successfully used these cells previously in screens as they have a well-defined LSC population and initiate AML with short latency, enabling rapid follow-up experiments in syngeneic hosts. ${ }^{12-15}$

Primitive NBM cells and MLL-AF9 leukemic cells were enriched by c-Kit selection, whereafter we assessed the effect of 114 murine cytokines in mixed cultures (Figure 1a and Supplementary Table 1). We used a cytokine concentration of $100 \mathrm{ng} / \mathrm{ml}$, as it has previously been used in similar screens. ${ }^{16}$ As a proof of principle, the screen identified several cytokines as selective positive regulators of leukemia cells previously associated with leukemic stem and progenitor cell biology, including CXCL12a (SDF1), IL3, tumor necrosis factor $a$ and IL6 (Figure 1b). ${ }^{6,7,17,18}$ In addition, several selective negative regulators of $A M L$ cells were identified and, of these, IL4 elicited the strongest depletion of leukemia cells (Figure 1b). Validation experiments demonstrated that the negative effect of IL4 on leukemia cell expansion was observed even in the presence of cytokines such as IL3, which gives strong proliferative signals, and without NBM cells present (Figure 1c). Moreover, IL4 inhibited colony-forming leukemia cells (Supplementary Figure 1). By contrast, IL4 did not affect the growth and survival of primitive NBM cells (Figure 1d).

IL4 induces apoptosis of AML cells in a p53-independent manner To explore the biological mechanism whereby IL4 exerts its negative effect on leukemia cells, we stimulated both $\mathrm{c}-\mathrm{Kit}^{+} \mathrm{MLL}-$ AF9 AML and c-Kit ${ }^{+}$NBM cells with IL4 and performed RNA sequencing. IL4 induced distinct gene expression signatures in the two cell populations (Figure $2 \mathrm{a}$ and Supplementary Figure $2 \mathrm{a}$ and b). To link the IL4-induced gene expression patterns in AML cells to previously reported gene expression signatures, we carried out gene set enrichment analysis. ${ }^{19}$ We found that the IL4 signature in leukemia cells was enriched for genes involved in cell death and Caspase pathway signatures (Figure $2 \mathrm{~b}$ and Supplementary Tables 3 and 4). Consistent with these findings, IL4 stimulation forced the AML cells into apoptosis as evident by an increase in Annexin $\mathrm{V}^{+}$cells (Figure $2 \mathrm{c}$ and Supplementary Figures $3 a$ and $b$ ), whereas their cell cycle or differentiation status was not affected (Figure $2 \mathrm{~d}$ and Supplementary Figures $3 \mathrm{c}$ and $\mathrm{d}$ ). Interestingly, by sorting the top $10 \%$ and lowest $10 \%$ IL4raexpressing cells and exposing them to IL4, we found that a higher IL4ra expression level sensitized the AML cells for the antileukemic effects of IL4 (Supplementary Figures $4 a-c$ ). By contrast, IL4 stimulation of NBM cells did not induce a gene expression signature that was enriched in any of the gene sets that matched with the IL4 signature in AML cells (false discovery rate $<0.05$; Supplementary Table 3). Moreover, we assessed which components of the IL4R complexes that are expressed in ${\mathrm{c}-\mathrm{Kit}^{+}}^{+}$leukemia cells, and found that the I/4ra and $1 / 2 \mathrm{rg}$ are expressed, but not I/13ra1 (Supplementary Figure 5).

To address whether IL4-induced apoptosis is mediated by p53, a well-known regulator of apoptosis, ${ }^{20}$ we used murine $T p 53^{-/-}$ MLL-AF9 leukemia cells, previously generated by us. ${ }^{13}$ We found that similar to $T p 53^{+/+}$AML cells (Figure $2 \mathrm{c}$ ), IL 4 forced $T p 53^{-/-}$ AML cells into apoptosis resulting in reduced cell numbers upon culture (Figures $2 \mathrm{e}$ and $\mathrm{f}$ ). These findings demonstrate that IL4 induces apoptosis of AML cells in a p53-independent manner.

IL4 induces apoptosis of AML cells in a Stat6-dependent manner To further explore the molecular mechanism underlying the antileukemic effect of IL4, we measured phosphorylation of Stat6, one of the downstream effectors of the IL4 receptor, ${ }^{21}$ upon IL4 treatment. We found that short exposure of leukemia cells to IL4 resulted in phosphorylation of Stat6 (Figure 3a). Moreover, Stat6target genes were enriched in the IL4 signature of AML cells as determined by gene set enrichment analysis (GSEA; Supplementary Table 3). To investigate whether the IL4-induced Stat6 activation was responsible for the depletion of leukemia cells, we first introduced ectopic Cas9 expression in the leukemia cells and sequentially a Stat6 sgRNA (Figure 3b). The sgRNA directed to Stat6 effectively suppressed Stat6 expression (Figures $3 c$ and d). To assess whether Stat6 disruption rendered the leukemia cells resistant to IL4, we monitored the percentage of sgRNA-expressing cells by tracking $\mathrm{GFP}^{+}$cells during IL4 stimulation. The leukemia cells that expressed the Stat6 sgRNA had a selective proliferative advantage upon IL4 treatment and exhibited reduced apoptosis, demonstrating that IL4 depletes the leukemia cells in a Stat6-dependent manner by inducing apoptosis (Figures $3 e$ and $\mathrm{f}$ ).

\section{IL4 selectively inhibits LSCs versus normal hematopoietic stem} cells

Following the identification of IL4 as a negative regulator of c-Kit ${ }^{+}$ AML cells in vitro, we next evaluated whether IL4 is also capable of inhibiting the growth and survival of leukemia-initiating cells. c-Kit ${ }^{+}$MLL-AF9 leukemia cells were cultured ex vivo for 3 days with or without IL4, and then transplanted into sublethally irradiated recipient mice. Notably, at 2 weeks after transplantation, we observed more than fourfold lower levels of leukemic cells in the peripheral blood of mice receiving IL4-treated cells, hereafter referred to as the IL4 group (Figure 4a). The IL4 groups survived longer than the control groups (Figures $4 \mathrm{~b}$ and $\mathrm{c}$ and Supplementary Figure 6), suggesting that IL4 inhibited leukemiainitiating cells. Moreover, we found that IL4 suppressed homing of the leukemia cells to the bone marrow as assessed $24 \mathrm{~h}$ post transplantation (Supplementary Figure 7). To evaluate whether IL4 affects normal hematopoietic stem and progenitor cell (HSPC) 
a

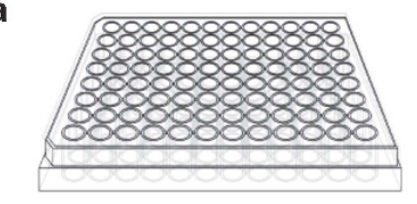

114 cytokines in triplicate $(100 \mathrm{ng} / \mathrm{mL})$
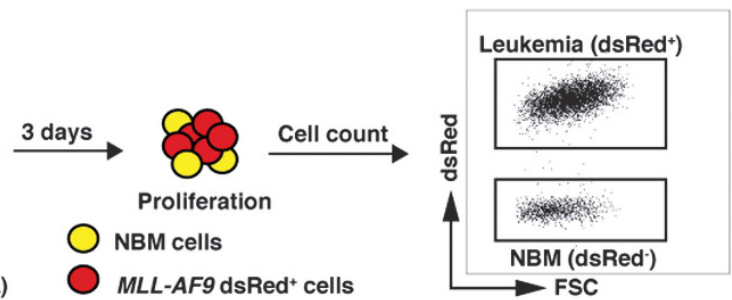

b
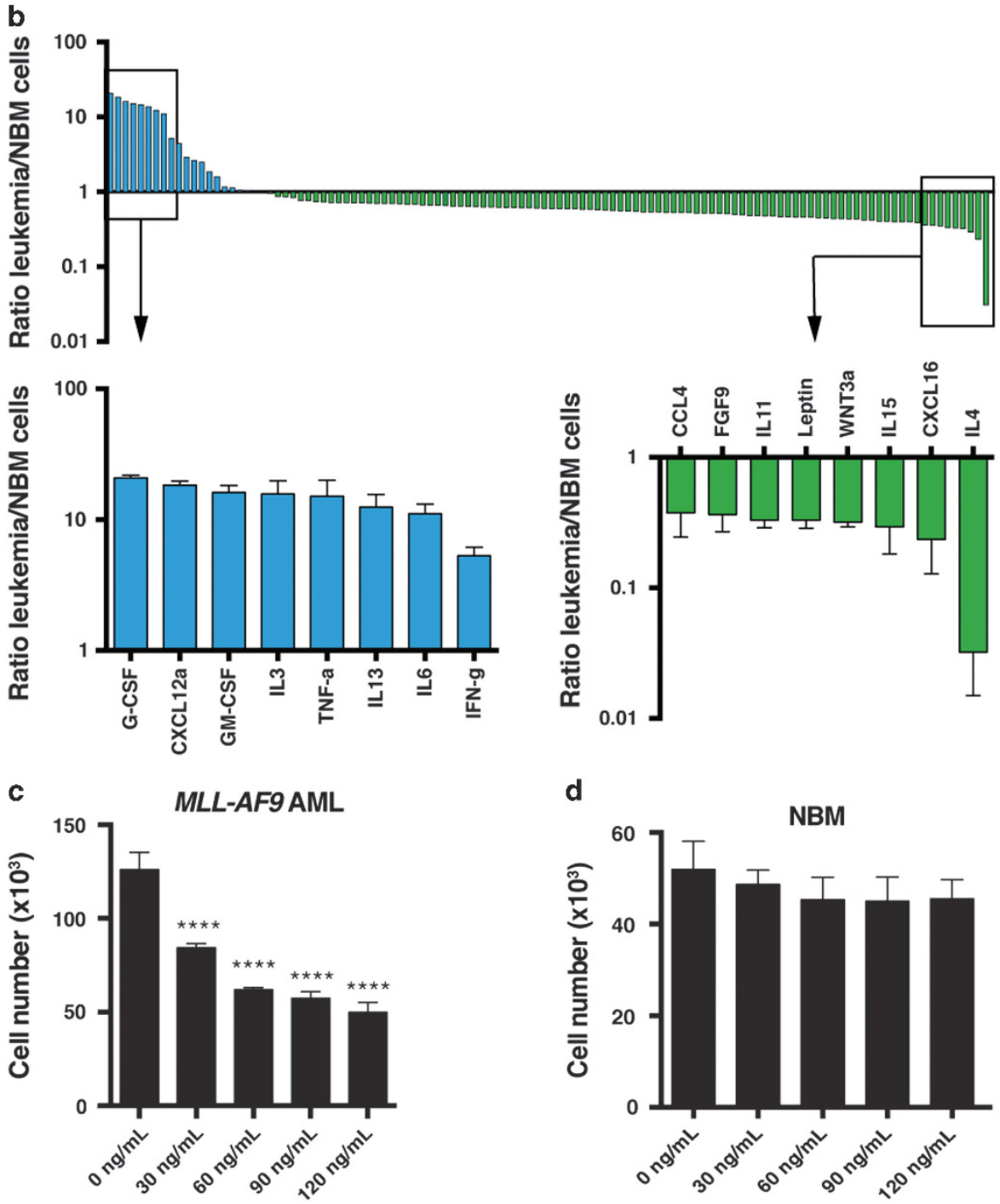

IL4 concentration

Figure 1. Cytokine screening identifies IL4 as a negative regulator of MLL-AF9 leukemia cells. (a) Schematic diagram showing the arrayed

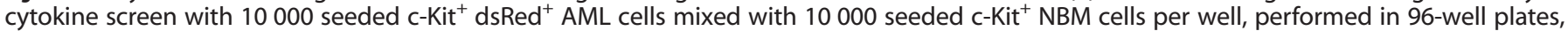
and without any baseline cytokines added to the medium. A cytokine library of 114 recombinant murine proteins was used, each tested separately. Cell number was determined using flow cytometry after 3 days of culture $(n=3)$. (b) Ranked screening results presented as the ratio in output cell number between leukemia $\left(\mathrm{dsRed}{ }^{+}\right.$) and NBM cells for each cytokine screened. Data from the full screen (blue, selective positive regulators of AML cells versus NBM cells; green, selective negative regulators of AML cells versus NBM cells). (c) Output cell number of 10000 seeded c-Kit ${ }^{+}$MLL-AF9 leukemia cells, and (d) $10000 \mathrm{c}-K i t^{+}$NBM cells, following dose titration with mlL4 for 3 days with mlL3 (20 ng/ml) as a baseline condition $(n=3)$. NBM, normal bone marrow. ${ }^{* * *} P<0.0001$.

function, we next cultured LSK bone marrow cells with or without IL4 and performed competitive bone marrow repopulation experiments (Supplementary Figure 8a). In contrast to the negative effect of IL4 on LSCs, IL4 treatment of normal HSPC cells did not significantly alter their short- or long-term bone marrow repopulating capacity, or affect their lineage fate (Supplementary Figures $8 b-e$ ).
IL4 is a negative regulator of AML cells in vivo

To address whether IL4 inhibits AML cells also in vivo, we generated a retroviral vector overexpressing mIL4 together with GFP (Figure 5a). Transductions of AML cells resulted in secretion of IL4 in the culture medium and about a 12-fold reduction in cell numbers upon 10-day cultures (Supplementary Figures 9a and b). 
a

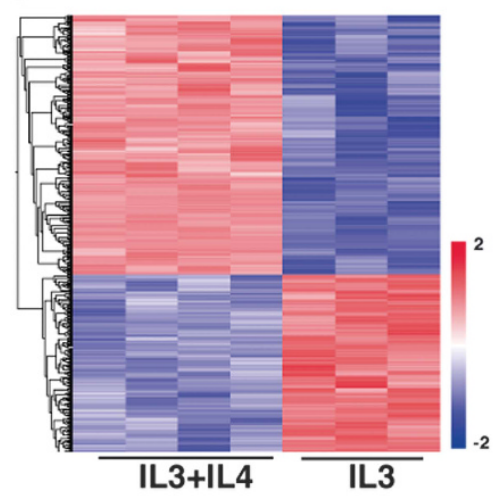

b

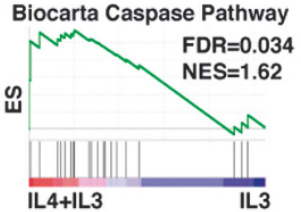

Biocarta Death Pathway

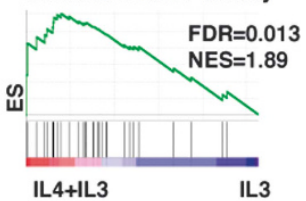

c

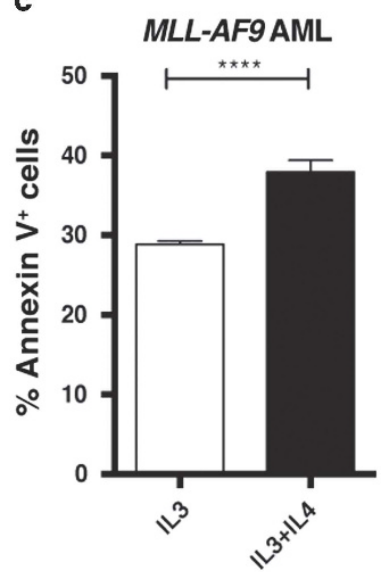

f

Tp53/AML d

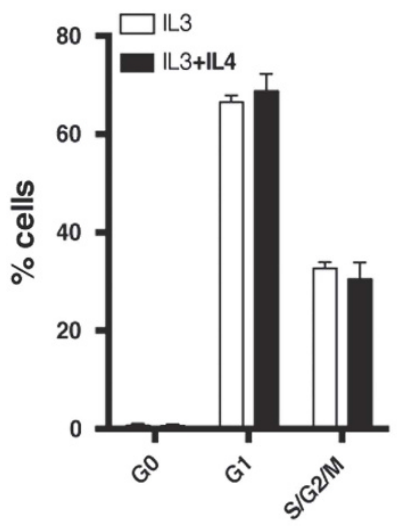

e

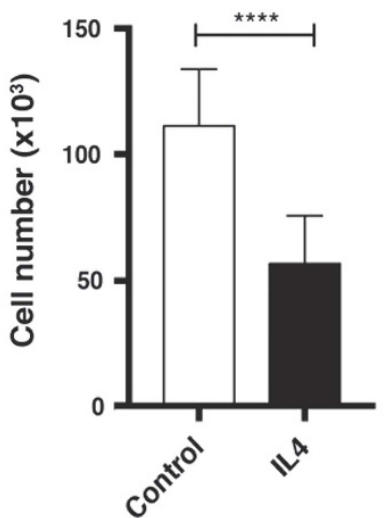

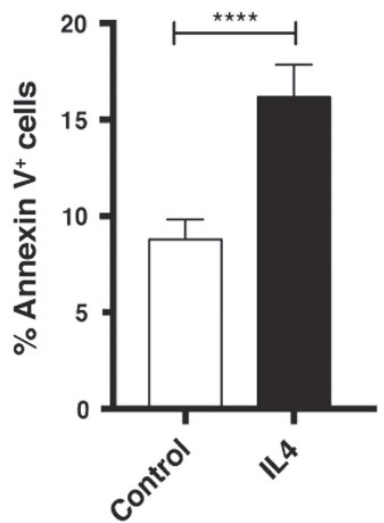

Figure 2. RNA sequencing reveals an IL4 signature in MLL-AF9 leukemia cells enriched for apoptosis-related gene expression. C-Kit ${ }^{+} M L L-A F 9$ AML cells were cultured with mIL4 and mIL3 or mIL3 only (control). (a) RNA sequencing of murine AML cells stimulated for $18 \mathrm{~h}$ with mIL4. A two-group comparison between control and IL4-treated AML cells revealed an IL4-induced signature of 390 differentially expressed genes (FDR $<0.05, P<0.001$ ). Genes in the heatmap are ordered by hierarchical clustering. (b) Gene set enrichment analysis (GSEA) showing that the IL4 gene expression signature in AML cells is enriched for Caspase and death pathways (Biocarta). (c) Apoptosis and (d) cell cycle analysis of leukemia cells treated with mIL4 for 3 days $(n=3)$. (e) Output cell number of 10000 seeded c-Kit ${ }^{+} T_{p 53^{-}}{ }^{-}$MLL-AF9 leukemia cells treated with mIL4 or control for 3 days $(n=3)$. (f) Apoptosis analysis of $c-K_{i t}^{+} T p 53^{-/-}$MLL-AF9 leukemia cells treated with mIL4 or control for 3 days $(n=3)$. ES, enrichment score; FDR, false discovery rate; NES, normalized enrichment score. ${ }^{* * *} P<0.0001$.

Next, we tested whether constitutive IL4 expression had a negative effect on AML cells also in vivo by transplanting sorted $\mathrm{GFP}^{+}$leukemia cells secreting IL4 shortly after transduction. At 2 weeks after transplantation, mice injected with IL4-secreting leukemia cells had elevated IL4 serum levels and reduced levels of leukemia cells in peripheral blood (Supplementary Figures 9c and d). The IL4 group survived significantly longer than the controls (median 38 versus 29 days, $P<0.0001$; Figure $5 \mathrm{~b}$ ). At the time of killing, mice in the IL4 group were almost devoid of leukemia cells in the bone marrow (mean $1.8 \%$ in the IL4 group versus $97 \%$ in the control group, $P<0.0001$; Figure $5 \mathrm{c}$ ) and spleen (mean $3.8 \%$ in the IL4 group versus $76 \%$ in the control group, $P<0.0001$; Supplementary Figure 9e), and had elevated levels of IL4 in the bone marrow (Supplementary Figure 9f), demonstrating that IL4 had strong antileukemic effects on AML cells in an in vivo context. Moreover, mice in the IL4 group had splenomegaly with an increase in $\mathrm{CD}^{+} \mathrm{T}$ cells, consistent with a role of IL4 in promoting $\mathrm{T}$ helper cells (Figure $5 \mathrm{~d}$ and Supplementary Figures $9 \mathrm{~g}$ and $\mathrm{h}$ ). ${ }^{22}$

We next explored whether intraperitoneal injections of IL4 into mice engrafted with AML cells would have antileukemic activity. IL4 injections into mice engrafted with AML cells resulted in lower leukemia burden in both blood (Figure 6a) and bone marrow
(Figure $6 \mathrm{~b}$ ), accompanied by a significantly prolonged survival (median 26 versus 23 days, $P<0.01$; Figure $6 c$ ). In contrast, upon IL4 injections into healthy mice, no lineage-skewing effects on hematopoietic cells were noted (Supplementary Figure 10). In summary, these findings demonstrate that intraperitoneal injections of IL4 also have a selective antileukemic effect on murine MLL-AF9 AML cells.

\section{IL4 induces apoptosis of human AML cells}

To assess whether IL4 has antileukemic activity also in human AML cells, we first stimulated the cytokine-dependent MA9 cell line ${ }^{23}$ with IL4. Similar to the effects we observed on murine MLL-AF9 AML cells, IL4 stimulation of MA9 cells resulted in suppression of cell proliferation (Supplementary Figure 11a), increased apoptosis via Caspase-3 activation and STAT6 phosphorylation (Figures 7a and $\mathrm{b}$ and Supplementary Figure 11b). Moreover, IL4 inhibited the colony formation of the MA9 cells (Supplementary Figure 11c). We next assessed which components of the IL4R complexes that are expressed in AML patient cells using RNA-sequencing data from TCGA. ${ }^{24}$ By contrast to the murine leukemia cells that expressed I/4ra and I/2rg only, we found that all three receptors are expressed in AML patient cells (Supplementary Figure 12). 
a

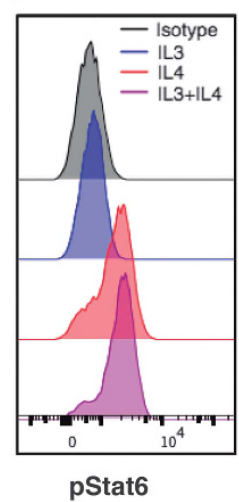

b

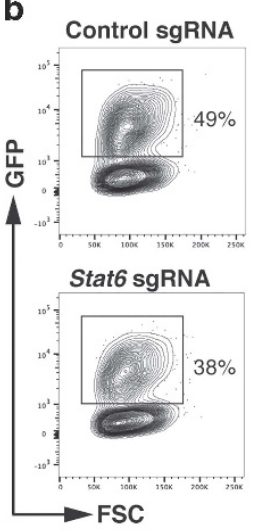

C

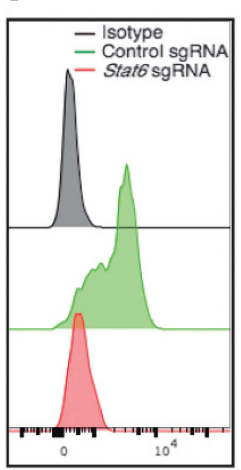

Total Stat6 d

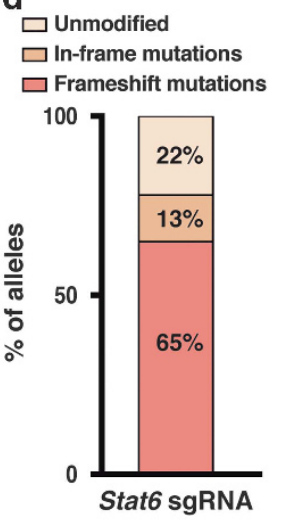

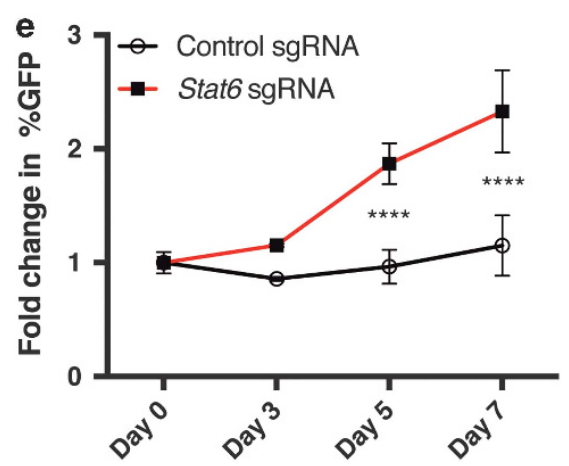

f

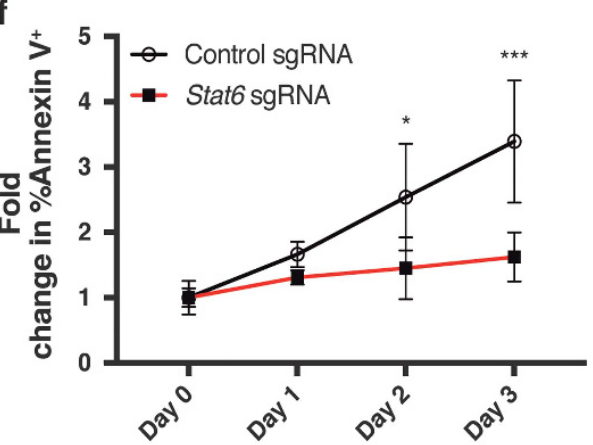

Figure 3. IL4 induces apoptosis of AML cells in a Stat6-dependent manner. (a) Staining of phosphorylated Stat6 (pStat6) upon stimulation with indicated cytokines in MLL-AF9 leukemia cells using flow cytometry. (b) Green fluorescent protein (GFP) expression in Cas9 ${ }^{+}$MLL-AF9 leukemia cells following transduction with lentiviral vectors coexpressing Stat6 or control sgRNAs and GFP. (c) Stat6 expression within GFP ${ }^{+}$cells 7 days post transduction. (d) Genetic alterations in Stat6 detected by next-generation sequencing within sorted GFP ${ }^{+}$cells 3 days post transduction. (e) Fold change in the percentages of GFP ${ }^{+}\left(\mathrm{sgRNA}^{+}\right)$cells within each group during mIL4 treatment relative to day 0 ( $n=3$ ). (f) Fold change in the percentages of Annexin $\mathrm{V}^{+}$cells within each group during IL4 treatment relative to day $0(n=3)$. ${ }^{*} P<0.05,{ }^{* * *} P<0.001,{ }^{* * * *} P<0.0001$.
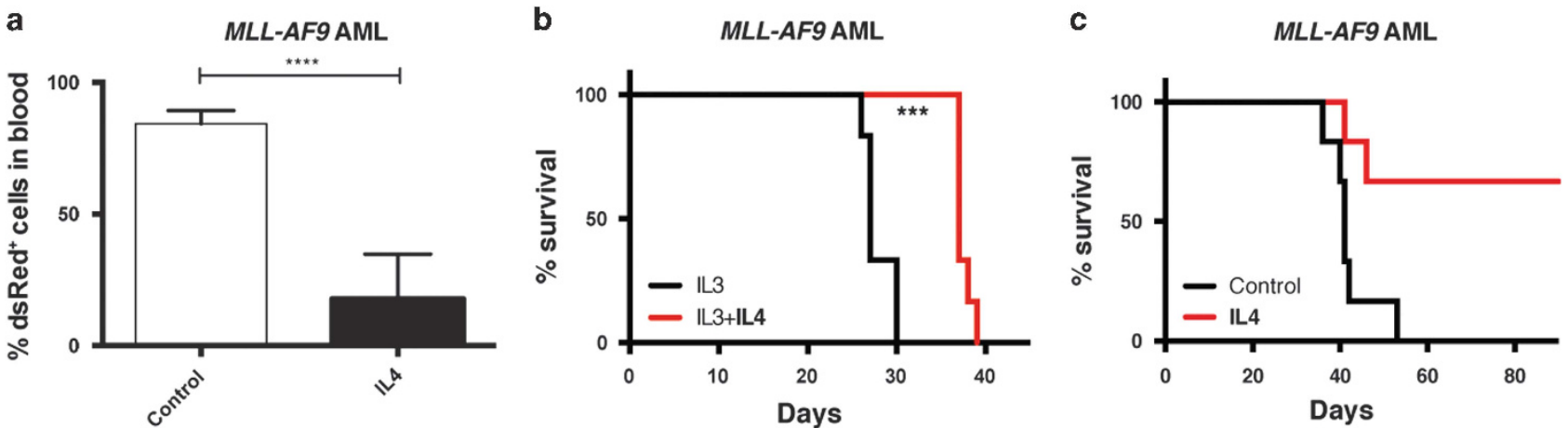

Figure 4. IL4 inhibits leukemia-initiating cells. (a, b) Overall, $500000 \mathrm{c}-K i t^{+}$MLL-AF9 AML cells were cultured ex vivo with mIL4 and mIL3 or mIL3 only (control) for 3 days, and then all treated cells were transplanted into sublethally irradiated mice (six mice per group). (a) Percentage of leukemic $\left(\mathrm{dsRed}^{+}\right)$cells in peripheral blood 14 days after transplantation. (b) Kaplan-Meier curves showing the survival of the mice. (c) In all,

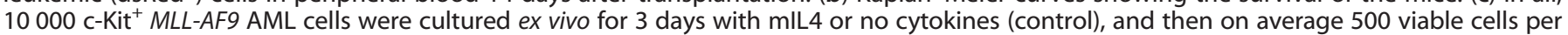
mouse were transplanted into sublethally irradiated mice for both groups (six mice per group). The Kaplan-Meier curves show the survival of the mice. ${ }^{* * *} P<0.001,{ }^{* * *} P<0.0001$.

We then evaluated whether IL4 has selective antileukemic activity also in primary AML patient cells relative to NBM cells. A majority of the patient samples tested had a normal karyotype, one had an MLL rearrangement and several harbored genetic alterations in FLT3 or NPM1 (Supplementary Table 2). In short-term cultures ( 3 days), IL4 treatment resulted in a significant decrease in cell number in six out of eight AML patient samples evaluated (Figure 7c). Similar to the effect observed in the murine AML model and the MA9 cell line, IL4 treatment induced apoptosis of primary AML patient cells (Figure 7d). By contrast, only one out of six NBM CD34 ${ }^{+}$samples responded to IL4 treatment (Figure 7e). Finally, we assessed the effect of IL4 on CD34 ${ }^{+} \mathrm{CD} 38^{-}$AML and NBM cells (Figure 7f), enriched for LSCs and normal hematopoietic stem cells, respectively. ${ }^{25,26}$ We found that IL4 inhibited the growth and survival of $\mathrm{AML} \mathrm{CD} 34^{+} \mathrm{CD} 38^{-}$cells in four out of six samples, whereas a milder effect of IL4 was detected on corresponding NBM samples (Figure $7 \mathrm{~g}$ ). These findings show that IL4 induces apoptosis and inhibits cell proliferation in a large 
a

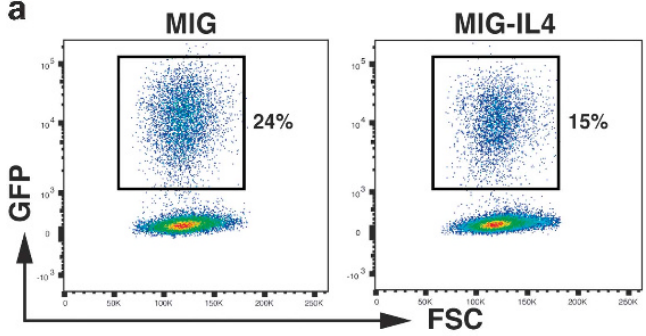

b
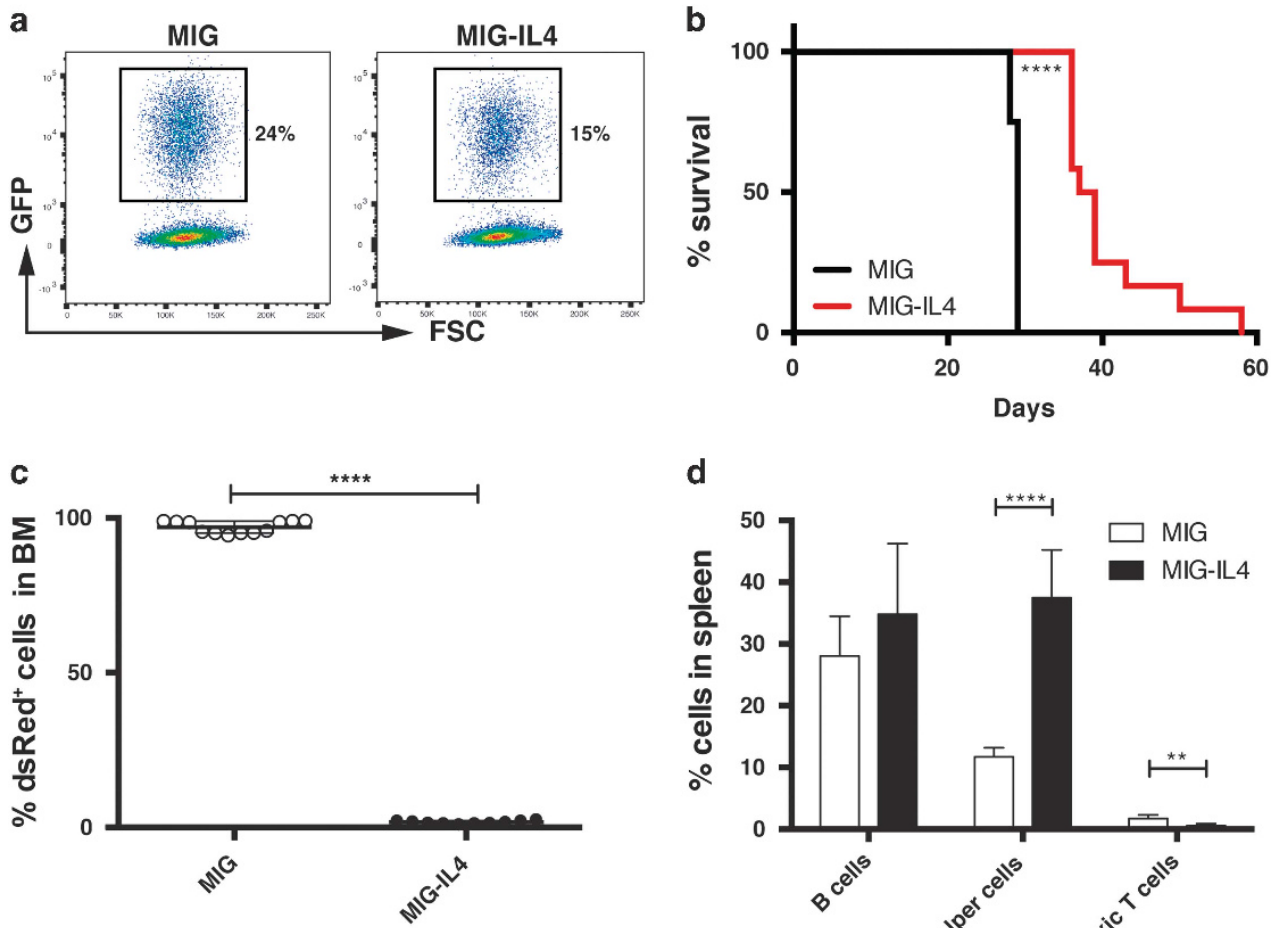

d

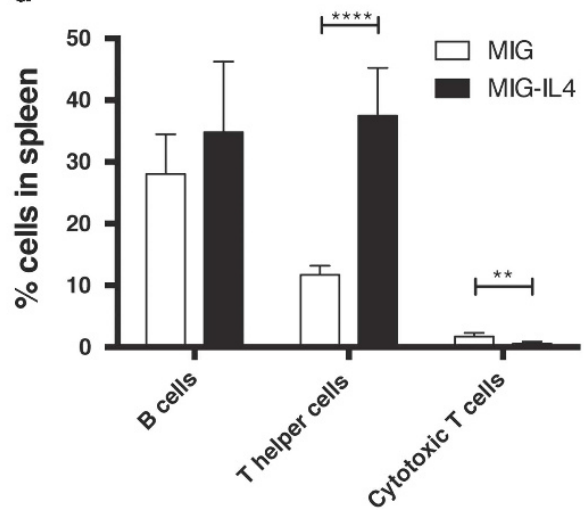

Figure 5. Ectopic expression of IL4 in AML cells in vivo has antileukemic effect. c-Kit ${ }^{+}$MLL-AF9 AML cells were transduced with retroviral vectors coexpressing a mIL4 cDNA (MIG-IL4) or a control vector (MIG) and GFP. Two days later, 30000 sorted GFP ${ }^{+}$cells were transplanted into sublethally irradiated mice. (a) GFP expression 2 days post transduction with the MIG and MIG-IL4 vectors. (b) Kaplan-Meier survival curves (12 mice per group). (c) Percentage of leukemia (dsRed ${ }^{+}$) cells in the bone marrow (BM) of mice at the time of killing. (d) Percentage of B cells $\left(B 220^{+}\right)$, T helper cells $\left(\mathrm{CD3}^{+} \mathrm{CD}^{+}\right)$and cytotoxic T cells $\left(\mathrm{CD}^{+} \mathrm{CD}^{+}\right)$within dsRed ${ }^{-}$(non-leukemic) cells in spleens of mice transplanted with IL4-secreting leukemia cells or controls (six mice per group). ${ }^{* *} P<0.01,{ }^{* * * *} P<0.0001$.
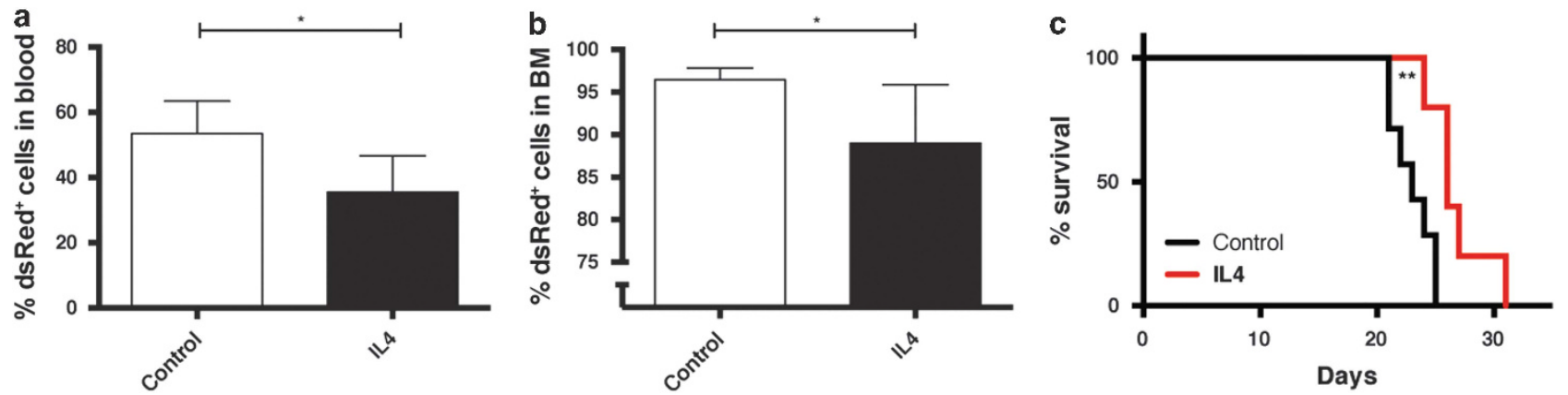

Figure 6. IL4 injections into mice suppresses leukemia burden and increases survival. Daily intraperitoneal injections of $\mathrm{mlL} 4(60 \mu \mathrm{g} / \mathrm{kg}$

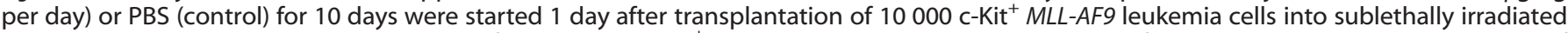
mice (six mice per group). (a) Percentage of leukemia (dsRed ${ }^{+}$) cells in peripheral blood on day 12 after transplantation. (b) Percentage of leukemia $\left(\mathrm{dsRed}^{+}\right)$cells in bone marrow $(\mathrm{BM})$ on day 13 after transplantation. (c) Kaplan-Meier curves showing overall survival of the mice. ${ }^{*} P<0.05,{ }^{* *} P<0.01$.

proportion of AML patient samples tested, whereas human NBM cells were less affected.

\section{DISCUSSION}

In a leukemic bone marrow, the deregulation of cytokines contributes to an alteration in bone marrow niche architecture, which contributes to leukemic progression, and suppression of normal hematopoiesis. ${ }^{3,27-30}$ We here established a cytokine screen using murine fluorescently labeled MLL-AF9 AML cells and identified IL4 as a selective negative regulator of primitive
AML cells. IL4 inhibited the growth and survival of MLL-AF9 AML cells both in cell cultures and in vivo without affecting normal HSPCs. IL4 is mainly known as an anti-inflammatory cytokine that binds to monocytes and suppresses the secretion of proinflammatory cytokines, such as IL1 and tumor necrosis factor- $a^{31-33}$ but it also has proinflammatory functions. ${ }^{34}$ Although IL4 has been described to have both positive and negative effects on cancer cells, ${ }^{35}$ the mechanistic basis for the IL4-induced negative effects on cancer cells remains obscure. ${ }^{36-39}$ Our finding that IL4 induces Caspase-3-mediated apoptosis of AML cells is in agreement with a previously described role for IL4 in inducing apoptosis of 

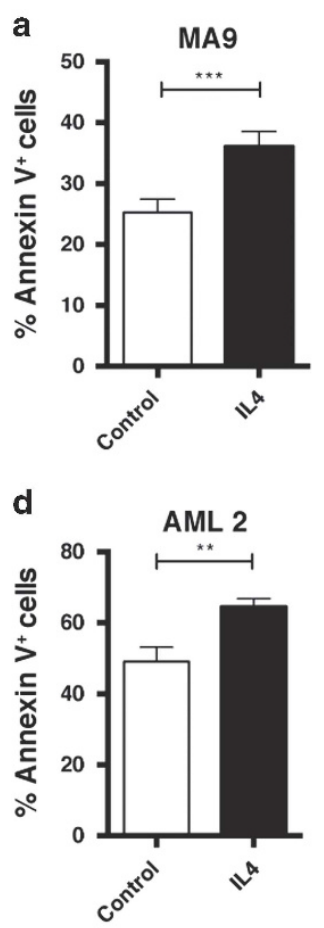

f $\quad$ AML 6

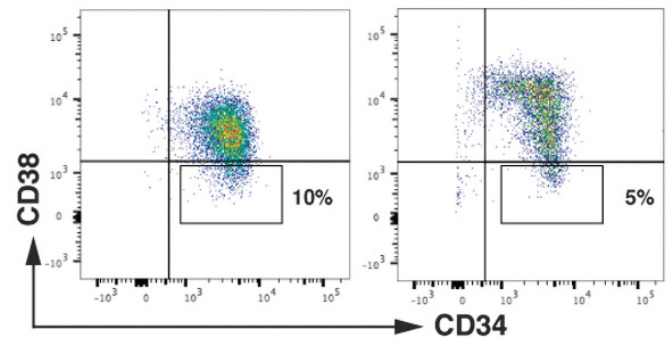

b

NBM 8
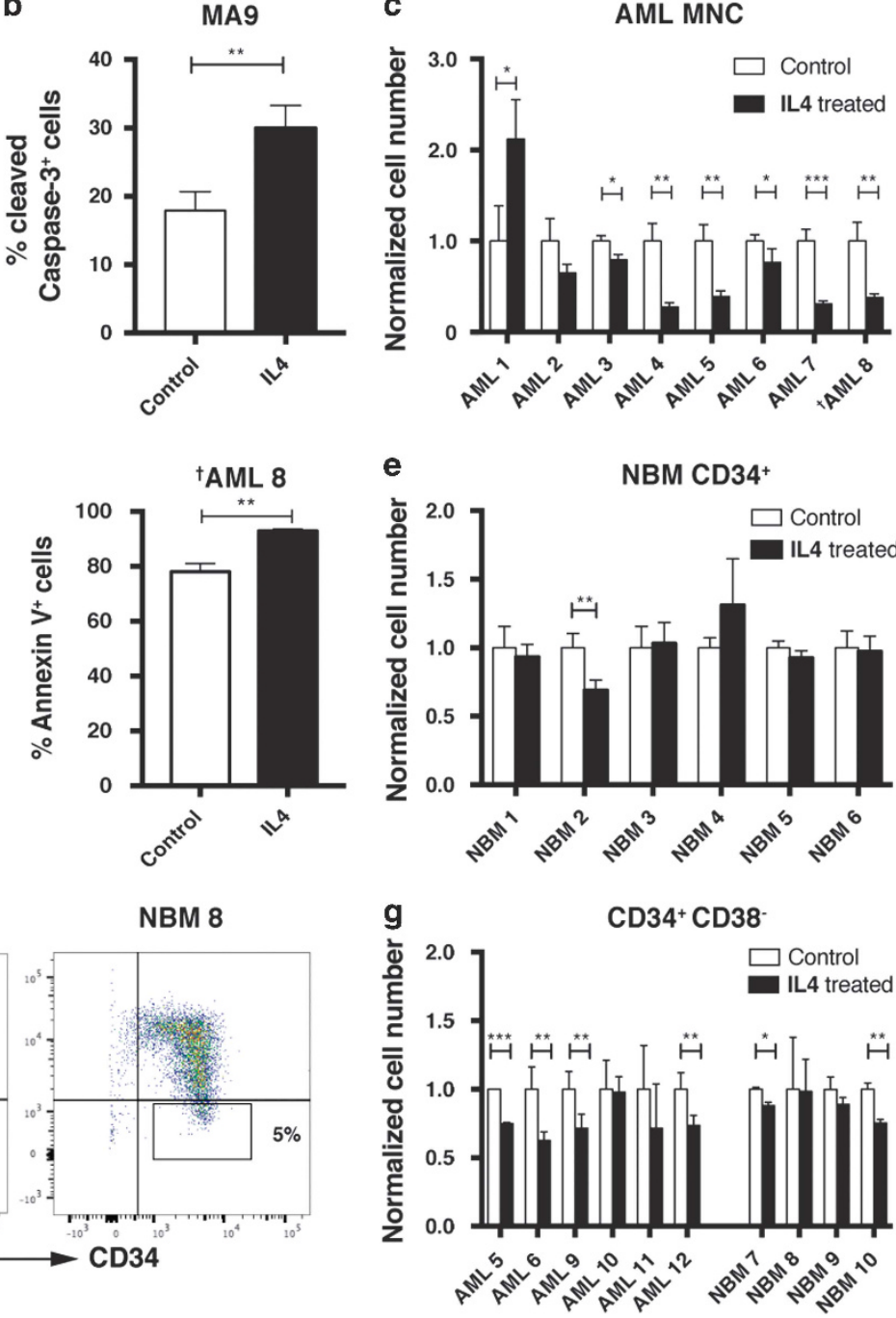

Figure 7. IL4 inhibits human AML cells by inducing apoptosis. Human leukemia cells were cultured for 3 days with hIL4 or without hIL4 (control). (a-b) MA9 leukemia cells treated with hIL4. (a) Annexin V and (b) Caspase-3 staining of MA9 cells $(n=3)$. (c-e) Primary AML mononuclear cells (MNCs) from patients and NBM CD34 ${ }^{+}$cells were treated with hIL4 $(n=3)$. (c) Output cell number of seeded AML MNCs following IL4 treatment. Output cell number is normalized to the control within samples. (d) Apoptosis analysis of MNCs from two AML samples (AML 2 and 8). (e) Output cell number of seeded NBM CD34 ${ }^{+}$cells following IL4 treatment. Output cell number is normalized to the control within samples. (f) FACS plots showing CD34 and CD38 staining of representative human CD34 ${ }^{+}$AML and NBM samples. Sorting of $\mathrm{CD} 34^{+} \mathrm{CD} 38^{-}$cells was performed according to the depicted gates. (g) Output cell number of seeded CD34 $4^{+} \mathrm{CD} 38^{-} \mathrm{AML}$ and NBM cells following treatment with hIL4. Output cell number is normalized to the control within samples. ${ }^{*} P<0.05$, ${ }^{* *} P<0.01,{ }^{* * *} P<0.001$. ${ }^{\dagger} P a t i e n t$ with an MLL-AF9 fusion gene.

monocytes. ${ }^{40}$ However, the finding that IL4 did not alter the cell cycle status of AML cells differs with observations in CLL cells, in which IL4 blocks cell cycle progression into the G1 stage. ${ }^{41}$

Consistent with Stat6 being a downstream mediator of IL4 signaling, ${ }^{21}$ we found that the antileukemic effect of IL4 in AML cells was, at least partially, Stat6-dependent. A similar link between IL4-induced apoptosis and Stat6 has previously been reported in breast cancer, but not in AML. ${ }^{42}$

Ex vivo IL4 treatment of leukemia cells suppressed the leukemiainitiating capacities of the cells, partially by inhibiting homing to the bone marrow, suggesting a negative effect of IL4 on LSCs. By contrast, the addition of IL4 to ex vivo cultures of normal HSPCs followed by competitive transplantations into mice did not reveal any effect on short- or long-term bone marrow repopulation, nor did it alter the lineage fate of the cells. These findings are in accordance with Stat6-deficient hematopoietic stem cells, which are functionally intact, even when challenged in competitive bone marrow repopulation assays. ${ }^{43}$

Importantly, IL4 inhibited AML cells also in vivo, with the most prominent antileukemic effect obtained upon retroviral secretion of IL4 by leukemic cells, as compared with intraperitoneal injections of IL4. Most likely, the stronger antileukemic effect obtained upon ectopic secretion of IL4 in vivo was attributed to a higher local concentration of IL4 using this strategy. However, because mice in the IL4 group at the time of killing did not have a fully developed leukemia, our data indicate poor tolerability of IL4 overexpression in vivo, potentially related to an expansion of $\mathrm{T}$ helper cells leading to aberrant cytokine secretion and splenomegaly. The expansion of $\mathrm{CD}^{+}{ }^{+} \mathrm{T}$ cells was not observed upon intraperitoneal injections of IL4, suggesting that the antileukemic effects of IL4 can be achieved within tolerable doses. 
Because IL4 inhibited the growth and survival of human colony-forming MA9 cells and the majority of AML patient samples tested, across several AML genetic and French-American-British subtypes, our data show that the antileukemic effect of IL4 first identified in the mouse model can be translated to human AML. However, to enhance the therapeutic efficacy of IL4, identifying therapies that potentially act in synergy with IL4 are warranted.

In summary, we here demonstrate that IL4 is a negative regulator of primitive $\mathrm{AML}$ cells, one of the first cytokines to be identified in this role, and provide in vitro and in vivo evidence that IL4 has antileukemic activity in AML. We show that IL4 acts by inducing apoptosis of AML cells, and its antileukemic effect is dependent on Stat6. These results highlight IL4 as a suppressor of primitive AML cells, findings that may translate into new therapeutic opportunities in AML.

\section{CONFLICT OF INTEREST}

The authors declare no conflict of interest.

\section{ACKNOWLEDGEMENTS}

We thank Dr Benjamin Ebert, Brigham and Women's Hospital, Boston, for sharing the $\mathrm{dsRed}^{+}$MLL-AF9 leukemia cells. We thank Dr James Mulloy, University of Cincinnati, Cincinnati, for sharing the MA9 cells. We thank the following granting agencies for their support: the Swedish Cancer Society, the Swedish Childhood Cancer Foundation, the Swedish Research Council, the Åke Wiberg Foundation, FP7 Marie Curie, BioCARE, the Crafoord Foundation, the Gunnar Nilsson Cancer Foundation, the Royal Physiographic Society in Lund, and the Medical Faculty of Lund University.

\section{REFERENCES}

1 Jemal A, Siegel R, Ward E, Murray T, Xu J, Thun MJ. Cancer statistics, 2007. CA Cancer J Clin 2007; 57: 43-66.

2 Burnett A, Wetzler M, Lowenberg B. Therapeutic advances in acute myeloid leukemia. J Clin Oncol 2011; 29: 487-494.

3 Mirantes C, Passegue E, Pietras EM. Pro-inflammatory cytokines: emerging players regulating HSC function in normal and diseased hematopoiesis. Exp Cell Res 2014; 329: 248-254

4 Zeng Z, Shi YX, Samudio IJ, Wang RY, Ling X, Frolova $O$ et al. Targeting the leukemia microenvironment by CXCR4 inhibition overcomes resistance to kinase inhibitors and chemotherapy in AML. Blood 2009; 113: 6215-6224.

5 Majeti R, Chao MP, Alizadeh AA, Pang WW, Jaiswal S, Gibbs KD Jr et al. CD47 is an adverse prognostic factor and therapeutic antibody target on human acute myeloid leukemia stem cells. Cell 2009; 138: 286-299.

6 Reynaud D, Pietras E, Barry-Holson K, Mir A, Binnewies M, Jeanne M et al. IL-6 controls leukemic multipotent progenitor cell fate and contributes to chronic myelogenous leukemia development. Cancer Cell 2011; 20: 661-673.

7 Gallipoli P, Pellicano F, Morrison H, Laidlaw K, Allan EK, Bhatia R et al. Autocrine TNF-alpha production supports CML stem and progenitor cell survival and enhances their proliferation. Blood 2013; 122: 3335-3339.

8 Lapidot T, Sirard C, Vormoor J, Murdoch B, Hoang T, Caceres-Cortes J et al. A cell initiating human acute myeloid leukaemia after transplantation into SCID mice. Nature 1994; 367: 645-648.

9 Ågerstam H, Karlsson C, Hansen N, Sanden C, Askmyr M, von Palffy S et al. Antibodies targeting human IL1RAP (IL1R3) show therapeutic effects in xenograft models of acute myeloid leukemia. Proc Natl Acad Sci USA 2015; 112: 10786-10791.

10 Ågerstam H, Hansen N, von Palffy S, Sandén C, Reckzeh K, Karlsson C et al. IL1RAP antibodies block IL-1-induced expansion of candidate CML stem cells and mediate cell killing in xenograft models. Blood 2016; 128: 2683-2693.

11 Zhang B, Chu S, Agarwal P, Campbell VL, Hopcroft L, Jørgensen HG et al. Inhibition of interleukin-1 signaling enhances elimination of tyrosine kinase inhibitortreated CML stem cells. Blood 2016; 128: 2671-2682.

12 Miller PG, Al-Shahrour F, Hartwell KA, Chu LP, Järås M, Puram RV et al. In Vivo RNAi screening identifies a leukemia-specific dependence on integrin beta 3 signaling. Cancer Cell 2013; 24: 45-58.

13 Järås M, Miller PG, Chu LP, Puram RV, Fink EC, Schneider RK et al. Csnk1a1 inhibition has p53-dependent therapeutic efficacy in acute myeloid leukemia. $J$ Exp Med 2014; 211: 605-612.
14 Hartwell KA, Miller PG, Mukherjee S, Kahn AR, Stewart AL, Logan DJ et al. Nichebased screening identifies small-molecule inhibitors of leukemia stem cells. Nat Chem Biol 2013; 9: 840-848.

15 Puram RV, Kowalczyk MS, de Boer CG, Schneider RK, Miller PG, McConkey M et al. Core circadian clock genes regulate leukemia stem cells in AML. Cell 2016; 165: 303-316.

16 Karlsson C, Baudet A, Miharada N, Soneji S, Gupta R, Magnusson M et al. Identification of the chemokine CCL28 as a growth and survival factor for human hematopoietic stem and progenitor cells. Blood 2013; 121: 3838-3842.

17 Mohle R, Bautz F, Rafii S, Moore MA, Brugger W, Kanz L. The chemokine receptor CXCR-4 is expressed on CD34+ hematopoietic progenitors and leukemic cells and mediates transendothelial migration induced by stromal cell-derived factor- 1 . Blood 1998; 91: 4523-4530.

18 Jin L, Lee EM, Ramshaw HS, Busfield SJ, Peoppl AG, Wilkinson L et al. Monoclonal antibody-mediated targeting of CD123, IL-3 receptor alpha chain, eliminates human acute myeloid leukemic stem cells. Cell Stem Cell 2009; 5: 31-42.

19 Subramanian A, Tamayo P, Mootha VK, Mukherjee S, Ebert BL, Gillette MA et al. Gene set enrichment analysis: A knowledge-based approach for interpreting genome-wide expression profiles. Proc Natl Acad Sci USA 2005; 102: 15545-15550.

20 Kiraz Y, Adan A, Kartal Yandim M, Baran Y. Major apoptotic mechanisms and genes involved in apoptosis. Tumour Biol 2016; 37: 8471-8486.

21 Wick KR, Berton MT. IL-4 induces serine phosphorylation of the STAT6 transactivation domain in B lymphocytes. Mol Immunol 2000; 37: 641-652.

22 Paul WE, Zhu J. How are TH2-type immune responses initiated and amplified? Nat Rev Immunol 2010; 10: 225-235.

23 Wei J, Wunderlich M, Fox C, Alvarez S, Cigudosa JC, Wilhelm JS et al. Microenvironment determines lineage fate in a human model of MLL-AF9 leukemia. Cancer Cell 2008; 13: 483-495.

24 Ley TJ, Miller C, Ding L, Raphael BJ, Mungall AJ, Robertson A et al. Genomic and epigenomic landscapes of adult de novo acute myeloid leukemia. $N$ Engl J Med 2013; 368: 2059-2074.

25 Bhatia M, Wang JC, Kapp U, Bonnet D, Dick JE. Purification of primitive human hematopoietic cells capable of repopulating immune-deficient mice. Proc Nat Acad Sci USA 1997; 94: 5320-5325.

26 Eppert K, Takenaka K, Lechman ER, Waldron L, Nilsson B, van Galen P et al. Stem cell gene expression programs influence clinical outcome in human leukemia. Nat Med 2011; 17: 1086-1093.

27 Schepers K, Pietras EM, Reynaud D, Flach J, Binnewies M, Garg T et al. Myeloproliferative neoplasia remodels the endosteal bone marrow niche into a selfreinforcing leukemic niche. Cell Stem Cell 2013; 13: 285-299.

28 Plaks V, Kong N, Werb Z. The cancer stem cell niche: how essential is the niche in regulating stemness of tumor cells? Cell Stem Cell 2015; 16: 225-238.

29 Lim M, Pang Y, Ma S, Hao S, Shi H, Zheng Y et al. Altered mesenchymal niche cells impede generation of normal hematopoietic progenitor cells in leukemic bone marrow. Leukemia 2016; 30: 154-162.

30 Dong L, Yu W-M, Zheng H, Loh ML, Bunting ST, Pauly M et al. Leukaemogenic effects of Ptpn 11 activating mutations in the stem cell microenvironment. Nature 2016; 539: 304-308.

31 Hart PH, Cooper RL, Finlay-Jones JJ. IL-4 suppresses IL-1 beta, TNF-alpha and PGE2 production by human peritoneal macrophages. Immunology 1991; 72 : 344-349.

32 te Velde AA, Huijbens RJ, Heije K, de Vries JE, Figdor CG. Interleukin-4 (IL-4) inhibits secretion of IL-1 beta, tumor necrosis factor alpha, and IL- 6 by human monocytes. Blood 1990; 76: 1392-1397.

33 Hart PH, Vitti GF, Burgess DR, Whitty GA, Piccoli DS, Hamilton JA. Potential antiinflammatory effects of interleukin 4: suppression of human monocyte tumor necrosis factor alpha, interleukin 1, and prostaglandin E2. Proc Natl Acad Sci USA 1989; 86: 3803-3807.

34 Van Kampen C, Gauldie J, Collins SM. Proinflammatory properties of IL-4 in the intestinal microenvironment. Am J Physiol Gastrointest Liver Physiol 2005; 288: G111-G117.

$35 \mathrm{Li}$ Z, Chen L, Qin Z. Paradoxical roles of IL-4 in tumor immunity. Cell Mol Immunol 2009; 6: 415-422.

36 Vellenga E, de Wolf JT, Beentjes JA, Esselink MT, Smit JW, Halie MR. Divergent effects of interleukin-4 (IL-4) on the granulocyte colony-stimulating factor and IL-3-supported myeloid colony formation from normal and leukemic bone marrow cells. Blood 1990; 75: 633-637.

37 Akashi K, Harada M, Shibuya T, Eto T, Takamatsu Y, Teshima T et al. Effects of interleukin-4 and interleukin- 6 on the proliferation of CD34+ and CD34- blasts from acute myelogenous leukemia. Blood 1991; 78: 197-204.

38 Akashi K, Shibuya T, Harada M, Takamatsu Y, Uike N, Eto T et al. Interleukin 4 suppresses the spontaneous growth of chronic myelomonocytic leukemia cells. J Clin Invest 1991; 88: 223-230. 
39 Hallett MA, Venmar KT, Fingleton B. Cytokine stimulation of epithelial cancer cells: the similar and divergent functions of IL-4 and IL-13. Cancer Res 2012; 72: 6338-6343.

40 Doseff Al, Baker JH Jr., Bourgeois TA, Wewers MD. Interleukin-4-induced apoptosis entails caspase activation and suppression of extracellular signal-regulated kinase phosphorylation. Am J Respir Cell Mol Biol 2003; 29 (3 Pt 1): 367-374.

41 Luo HY, Rubio M, Biron G, Delespesse G, Sarfati M. Antiproliferative effect of interleukin-4 in B chronic lymphocytic leukemia. J Immunother 1991; 10: 418-425.

42 Gooch JL, Christy B, Yee D. STAT6 mediates interleukin-4 growth inhibition in human breast cancer cells. Neoplasia 2002; 4: 324-331.

43 Bunting KD, Yu WM, Bradley HL, Haviernikova E, Kelly-Welch AE, Keegan AD et al. Increased numbers of committed myeloid progenitors but not primitive hematopoietic stem/progenitors in mice lacking STAT6 expression. J Leuk Biol 2004; 76: 484-490.

(c) (i) This work is licensed under a Creative Commons Attribution 4.0 International License. The images or other third party material in this article are included in the article's Creative Commons license, unless indicated otherwise in the credit line; if the material is not included under the Creative Commons license, users will need to obtain permission from the license holder to reproduce the material. To view a copy of this license, visit http://creativecommons.org/licenses/by/4.0/

(c) The Author(s) 2018

Supplementary Information accompanies this paper on the Leukemia website (http://www.nature.com/leu) 